\title{
INTERFACE GRÁFICA PARA RESOLUÇÃO PASSO A PASSO DE SISTEMAS TRIFÁSICOS
}

Amanda M. P. Odate - amandaodatemp@gmail.com

Universidade Federal do Pará - UFPA, Faculdade de Engenharia Elétrica e Biomédica FEEB

R. Augusto Corrêa, 01

66075-110-Belém-Pará

Arthur F. S. Fernandes - arthurengl.ufpa@gmail.com

João V. M. Silva - victormourasilval@gmail.com

Luiz G. L. Costa-luizgustavo.ufpa@gmail.com

Orlando F.Silva-orfosi@ufpa.br

Resumo: Este trabalho apresenta o desenvolvimento de uma ferramenta gráfica para o estudo de sistemas trifásicos. A partir da observação das dificuldades e necessidades enfrentadas por estudantes inseridos na vida acadêmica em estudar tal assunto, verificou-se a possibilidade da elaboração dessa ferramenta com o intuito de facilitar a aprendizagem do tema abordado. Para tanto, utilizou-se o software MatLab para a criação de uma plataforma capaz de fazer cálculos de transformação de impedância, além de exibir o passo a passo do resultado. Com isso, espera-se que o estudo deste assunto se torne muito mais fluido e eficiente para o aluno.

Palavras-chave: Sistemas trifásicos. Software MatLab. Transformações de sistemas trifásicos equilibrados e desequilibrados. Ferramenta de aprendizagem em engenharia.

\section{INTRODUÇÃO}

Uma das maiores conquistas de engenharia do século XX foi a consolidação do uso da energia elétrica no cotidiano, elevando o padrão de vida de muitas populações e possibilitando a inovação de inúmeras tecnologias. Diante desse cenário, constatou-se que o sistema trifásico seria o mais conveniente por razões econômicas e técnicas como a transmissão de potência com o menor custo e com a utilização de motores de indução trifásicos. Assim, esse modelo de sistema passou a ser o padrão para a geração, transmissão e distribuição de energia em corrente alternada. (ROBBA, 1996)

Devido a sua relevância para o cotidiano da sociedade, é de suma importância o estudo e o aprimoramento dessa tecnologia. Com o objetivo de auxiliar os estudos em relação ao tema, foi desenvolvida uma plataforma gráfica, utilizando a ferramenta GUIDE do MatLab, que permite a conversão de tipos de ligação dos sistemas trifásicos, além de apresentar o passo-apasso da resolução. A ferramenta calcula automaticamente o que previamente seria feito à mão pelo estudante, facilitando a verificação dos cálculos feitos. Dessa forma, espera-se que a plataforma facilite a retenção do assunto abordado em sala de aula de forma mais eficiente. 


\section{FUNDAMENTAÇÃO TEÓRICA}

Para entendimento de como um sistema trifásico é gerado, pode-se considerar inicialmente um gerador monofásico, neste caso, nos terminais de uma bobina que gira com velocidade angular constante, no interior de um campo magnético uniforme, surge uma tensão senoidal. Se sobre o mesmo eixo forem dispostas três bobinas deslocadas entre si de $2 \pi / 3 \mathrm{rad}$ e o conjunto girado com velocidade angular constante no sentido horário, no interior de um campo magnético uniforme, nos terminais das bobinas aparecerá um sistema de tensões de mesmo valor máximo e defasadas entre si de $2 \pi / 3 \mathrm{rad}$ (Circuitos Trifásicos, 201-), conforme ilustra a Figura 1.

Figura 1

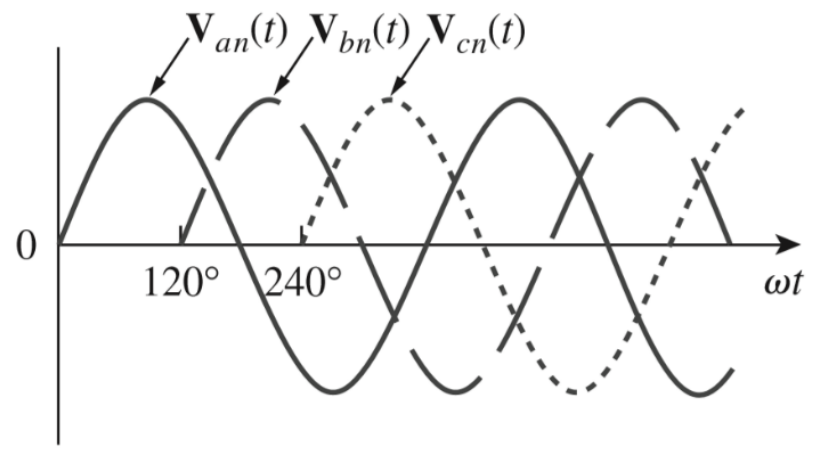

Fonte: Fundamentos de Circuitos Elétricos - SADIKU

Um sistema trifásico típico é formado por três fontes de tensão conectadas a cargas por três ou quatro fios. As fontes de tensão podem ser interligadas em triângulo ou em estrela, totalizando assim, quatro possíveis possibilidades de ligação. Além disso, esse sistema apresenta dois tipos de configuração, sistema com cargas equilibradas ou desequilibradas.

\subsection{Fasores}

Fasor é um número complexo que representa a amplitude e a fase de uma senoide (SADIKU, 2013). Fasores são ferramentas que representam funções sem a presença do tempo, facilitando as operações matemáticas a serem realizadas. Um fasor é composto por módulo e fase como mostra a Equação 1, onde z é um número complexo, r é o seu módulo e $\phi$ é a sua fase, a representação gráfica desta equação pode ser observada na Figura 2.

$$
z=r \angle \phi
$$


Figura 2-Gráfico de z

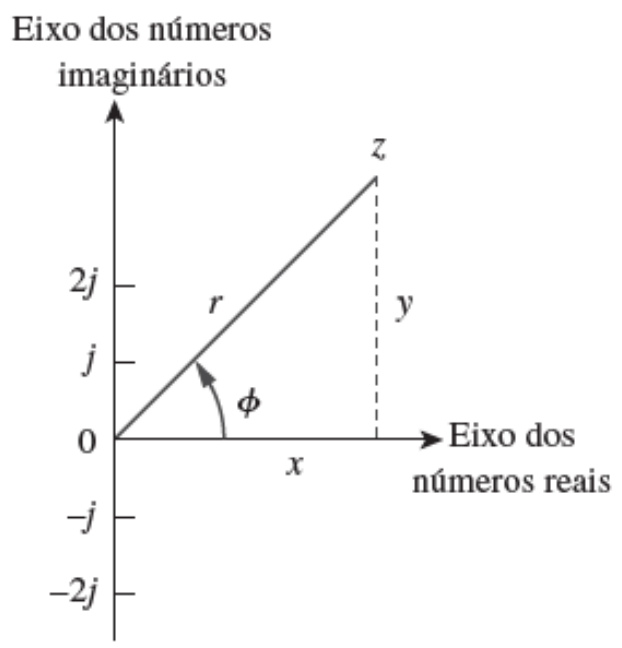

Fonte: Fundamentos de Circuitos Elétricos - SADIKU

O fasor $\alpha$ expresso na Equação 2, em particular, será especialmente útil para facilitar as operações. Este fasor é útil uma vez que existem duas formas de explicitar os valores trifásicos analisados: direta (positiva) e inversa (negativa).

$$
\alpha=1 \angle 120^{\circ}
$$

\subsection{Fontes e cargas trifásicas}

Uma fonte trifásica é composta, na realidade, por três fontes monofásicas, cada fonte atua independentemente, por isso, não é incomum um desequilíbrio entre as fontes. A associação das fontes pode ser feita de duas formas: estrela $(Y)$ e triângulo $(\Delta)$. As diferenças nas ligações são justificadas pela existência de duas medições: correntes e tensões de linha, são os valores medidos diretamente na fonte; correntes e tensões de fase, são os valores medidos nos terminais de saída.

Uma carga, independente da forma de associação, estará desequilibrada se as impedâncias por trilha não forem iguais em magnitude ou fase. Analogamente, uma fonte estará desequilibrada se as tensões ou as correntes por trilha não sejam iguais em magnitude e com a defasagem correta.

\section{Estrela}

Uma fonte trifásica genérica ligada em $Y$ é apresentada na Figura 3, a principal vantagem de realizar a ligação em estrela consiste no fato de que a corrente de linha é igual a corrente de fase, as Equações 3 a 9 regem o funcionamento da fonte em $Y$. 
Figura 3 - Fonte ligada em $Y$

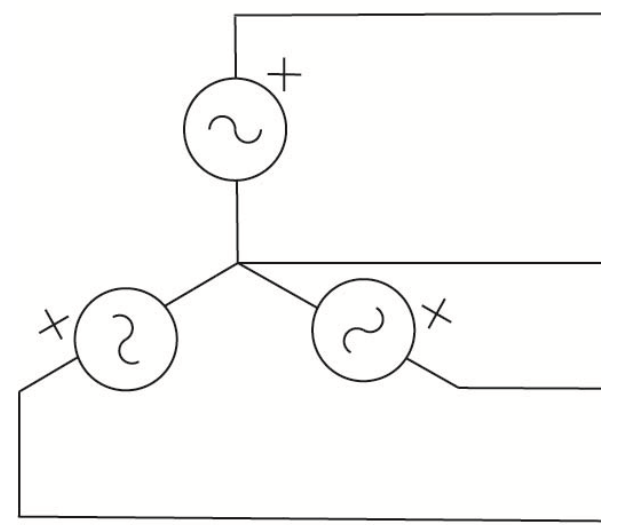

Fonte: Autores

$$
\begin{gathered}
V_{a b}+V_{b c}+V_{c a}=0 \\
V_{a b}=V_{a n}-V_{b n}=|V| \angle 0^{\circ}-|V| \angle-120^{\circ} \\
V_{b c}=V_{b n}-V_{c n}=|V| \angle-120^{\circ}-|V| \angle 120^{\circ} \\
V_{c a}=V_{c n}-V_{a n}=|V| \angle 120^{\circ}-|V| \angle 0^{\circ} \\
I_{\text {linh } a}=I_{\text {fase }}
\end{gathered}
$$

\section{Triângulo}

Uma fonte trifásica genérica ligada em $\Delta$ é apresentada na Figura 4, a principal vantagem de realizar a ligação em triângulo consiste no fato de que a tensão de linha é igual a tensão de fase, as Equações 8 a 12 regem o funcionamento da fonte em triângulo.

Figura 4-Fonte ligada em $\Delta$

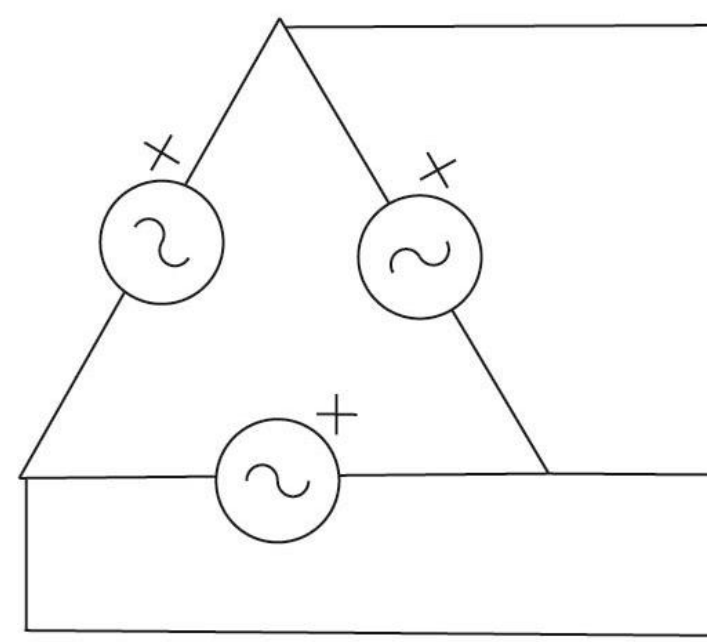

Fonte: Autores 


$$
\begin{gathered}
I_{a}+I_{b}+I_{c}=0 \\
I_{a}=I_{a b}-I_{c a}=|I| \angle 0^{\circ}-|I| \angle 120^{\circ} \\
I_{b}=I_{b c}-I_{a b}=|I| \angle-120^{\circ}-|I| \angle 0^{\circ} \\
I_{c}=I_{c a}-I_{b c}=|I| \angle 120^{\circ}-|I| \angle-120^{\circ} \\
V_{\text {linh } a}=V_{\text {fase }}
\end{gathered}
$$

\subsection{Fontes trifásicas}

As conexões entre fonte e carga podem ser feitas de 4 formas: $\mathrm{Y}-\Delta, \Delta-\mathrm{Y}, \Delta-\Delta, \mathrm{Y}-\mathrm{Y}$. Cada conexão possui seu próprio modelo de equações que a torna vantajosa em determinada aplicação, com exceção da Y-Y, que raramente é utilizada devido à problemas de natureza prática.

\section{Estrela-Triângulo}

$\mathrm{Na}$ Figura 5 tem-se a Conexão de fonte em Y e carga em $\Delta$.

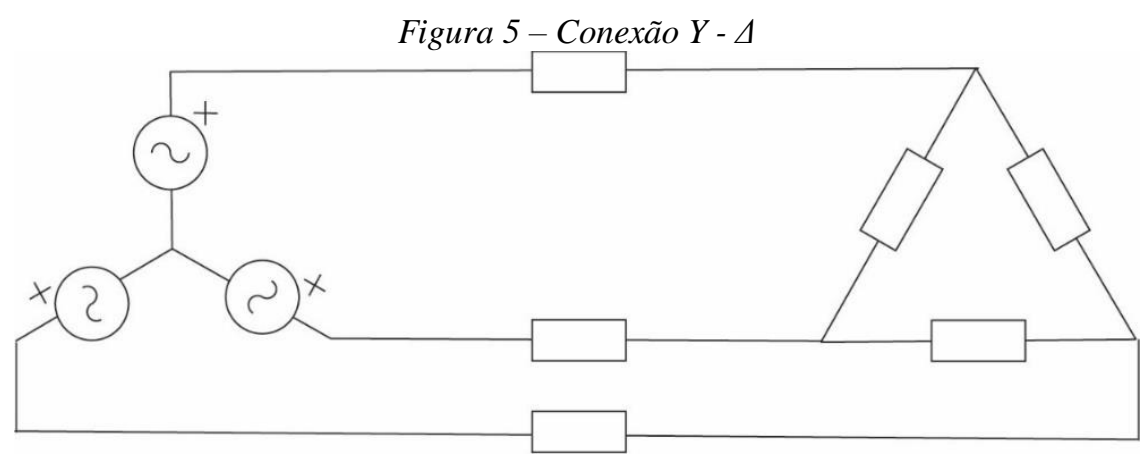

Fonte: Autores

\section{Triângulo-Estrela}

$\mathrm{Na}$ Figura 6 tem-se a Conexão de fonte em $\Delta$ e carga em Y.

Figura 6-Conexão $\Delta-Y$

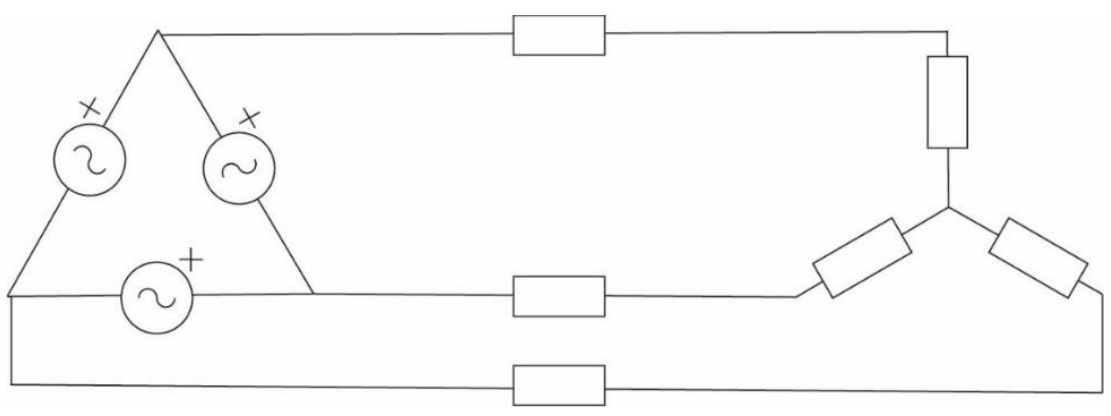

Fonte: Autores

\section{Triângulo-Triângulo}

$\mathrm{Na}$ Figura 7 tem-se a Conexão de fonte e carga em $\Delta$ em $\Delta$. 


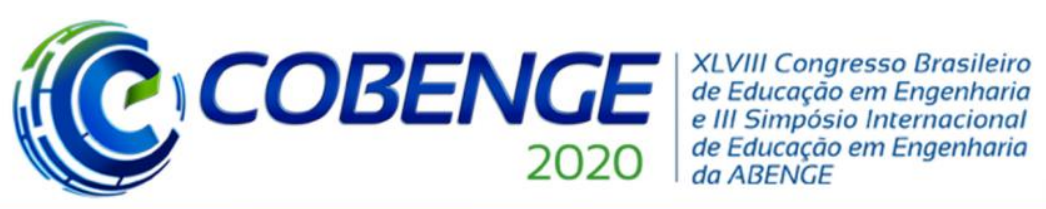

"Os desafios para formar hoje o engenheiro do amanhã"
01 a 03 de dezembro Evento On-line

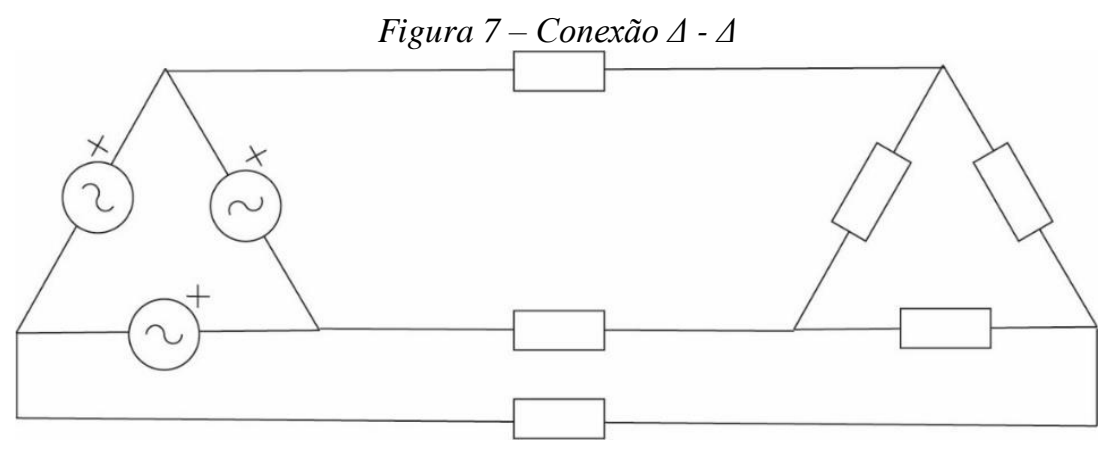

Fonte: Autores

\section{Estrela-Estrela}

Na Figura 8 tem-se a Conexão de fonte e carga em Y.

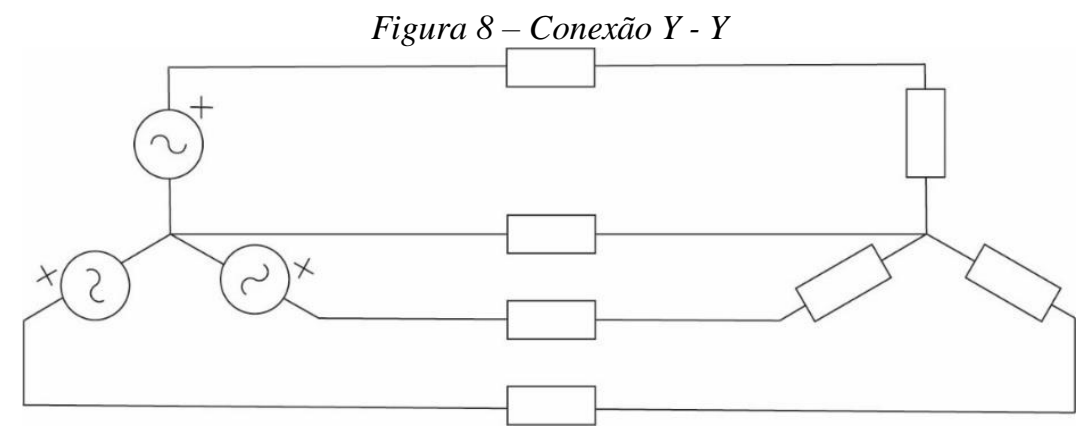

Fonte: Autores

\section{INTERFACE DESENVOLVIDA}

A seguinte plataforma foi elaborada de forma a contribuir com o processo de aprendizagem do discente. Após a aluno ser exposto às informações tratadas na fundamentação teórica, o conhecimento poderá ser retido utilizando para tal a ferramenta abordada. Ela ajudará no processo de solidificação do conhecimento devido à possibilidade de utilização de forma a ratificar os cálculos feitos à mão, além de exibir o passo a passo.

A ferramenta foi desenvolvida utilizando o aplicativo GUIDE (Matlab, 2010). Sua interface é ilustrada na Figura 9 e em seguida sua utilização é detalhada. 
Figura 11 - Menu carga

\begin{tabular}{l}
\hline configcarga \\
Impedância Za \\
Impedância Zb \\
Impedância Zc \\
Impedância da Linha \\
Impedância de Neutro \\
\\
\\
Saljo \\
Salvar \\
\hline
\end{tabular}

Fonte: Autores

Após preencher as informações de ambos os menus, clica-se em atualizar e seguidamente, clica-se no botão 'resultados' para observar de forma resumida as informações do circuito trifásico, como ilustra a Figura 12. Paralelamente, pode-se clicar no botão 'passoa-passo' para visualizar o procedimento necessário para atingir a resposta correta do problema.

Figura 12 - Menu resultados

\begin{tabular}{|c|c|c|}
\hline 4. resultado & - & $\square$ \\
\hline \multicolumn{3}{|c|}{ Resultados } \\
\hline -Tensões de Fase - & \multicolumn{2}{|c|}{-Tensões de Linha } \\
\hline$V a^{\prime} n^{\prime}=100.4 \mathrm{~V},-4.8^{\circ}$ & \multicolumn{2}{|c|}{$V a^{\prime} b^{\prime}=183.9 \mathrm{~V}, 26.8^{\circ}$} \\
\hline$V b^{\prime} n^{\prime}=111.5 \mathrm{~V},-125.2^{\circ}$ & \multicolumn{2}{|c|}{$V^{\prime} b^{\prime} c^{\prime}=169.3 \mathrm{~V},-89.3^{\circ}$} \\
\hline$V c^{\prime} n^{\prime}=102.5 \mathrm{~V}, 130.4^{\circ}$ & \multicolumn{2}{|c|}{$V c^{\prime} a^{\prime}=187.5 \mathrm{~V}, 152.5^{\circ}$} \\
\hline \multicolumn{3}{|c|}{- Correntes de Fase -- Correntes de Linha } \\
\hline $\mathrm{la}^{\prime} n^{\prime}=9.0 \mathrm{~A},-31.3^{\circ}$ & \multicolumn{2}{|c|}{$\begin{array}{c}\text { Correntes de Linha } \\
\text { la }=\quad 9.0 \mathrm{~A},-31.3^{\circ}\end{array}$} \\
\hline $\mathrm{lb}^{\prime} n^{\prime}=\quad 13.0 \mathrm{~A},-145.7^{\circ}$ & \multicolumn{2}{|c|}{$\mathrm{lb}=13.0 \mathrm{~A},-145.7^{\circ}$} \\
\hline$I C^{\prime} n^{\prime}=\quad 14.5 \mathrm{~A}, 48.5^{\circ}$ & \multicolumn{2}{|c|}{$I C=\quad 14.5 \mathrm{~A}, 48.5^{\circ}$} \\
\hline \multicolumn{3}{|c|}{ Tensão e Corrente do Neutro } \\
\hline$V n n=9.3 \mathrm{~V}, 34.9^{\circ}$ & $\begin{array}{c}\text { Neutro } \\
\text { In }=\end{array}$ & $6.6 \mathrm{~A},-10.1^{\circ}$ \\
\hline \multicolumn{3}{|l|}{ Potências } \\
\hline Complexa - & \multicolumn{2}{|c|}{-Aparente } \\
\hline$\overline{\mathrm{S}} \mathrm{a}=805.8+\mathrm{j} 402.9 \mathrm{VA}$ & & $S_{9}=900.9 \mathrm{VA}$ \\
\hline$\overline{\mathrm{S}} \mathrm{b}=1362.0+j 510.8 \mathrm{~V}$ & $\mathrm{Sb}=$ & $1454.6 \mathrm{VA}$ \\
\hline $\bar{S} \mathrm{c}=210.2+j 1471.6 \mathrm{~V}$ & $\mathrm{Sc}=$ & $1486.6 \mathrm{VA}$ \\
\hline - Ativa & \multicolumn{2}{|l|}{-Reativa } \\
\hline $805.8 \mathrm{~W}$ & $\mathrm{Qa}=$ & 402.9 VAR \\
\hline $1362.0 \mathrm{~W}$ & $\mathrm{Qb}=$ & 510.8 VAR \\
\hline $210.2 \mathrm{~W}$ & $Q C=$ & 1471.6 VAR \\
\hline
\end{tabular}


Fonte: Autores

\title{
4 CONSIDERAÇÕES FINAIS
}

Todos os requisitos almejados para o desenvolvimento dessa ferramenta foram alcançados e, após isso, a mesma seria apresentada junto a turma da disciplina que trabalha o conceito de circuitos de segunda ordem para uma avaliação mais ampla pelos seus principais usuários finais. A avaliação seria por meio de um questionário onde os principais objetivos seriam verificar: a aplicabilidade da ferramenta na disciplina, a contribuição da ferramenta no ensino aprendizagem da turma e a identificação de possíveis melhorias na ferramenta, no entanto, as atividades nas universidades foram suspensas devido a quarentena e a pandemia do COVID-19, o que inviabilizou temporariamente esta avaliação. Todos os requisitos almejados para o desenvolvimento dessa ferramenta foram alcançados e, após isso,

\section{REFERÊNCIAS}

Circuitos

Trifásicos.

Disponível

em: https://edisciplinas.usp.br/pluginfile.php/4707371/mod_resource/content/1/teo_circuitos_trifa sicos.pdf. Acesso em: 22 mai. 2020.

MATLAB. Matlab documentation. Disponível em: www.mathworks.com/help/matlab/. Acesso em: 18 abr. 2020.

ROBBA, Ernesto et al. Introdução a Sistemas Elétrico de Potência. $2^{a}$ ed. São Paulo: Editora Edgar Blücher LTDA. 1996.

SADIKU, M. N. O., ALEXANDER, C. K., Fundamentos de circuitos elétricos. $5^{\text {a }}$ edição. Porto Alegre: AMGH, 2013.

\section{GRAPHIC INTERFACE FOR STEP BY STEP RESOLUTION THREE-PHASE SYSTEMS}

\begin{abstract}
This work presents the development of a graphic tool for the study of three-phase systems. From the observation of the difficulties and needs faced by students inserted in the academic life in studying this subject, the possibility of developing this tool was verified in order to facilitate the learning of the topic addressed. For that, the MatLab software was used to create a platform capable of making calculations of impedance transformation, in addition to displaying the results step by step. Thus, it is expected that the study of this subject will become much more fluid and efficient for the student.
\end{abstract}

Keywords: Three-phase systems. MatLab software. Transformations of balanced and unbalanced three-phase systems. Engineering learning tool. 